\title{
Tangence
}

\section{Un air de famille. Entre La relève et Refus global : la génération cachée}

\section{Marcel Olscamp}

Numéro 62, avril 2000

Parentèle

URI : https://id.erudit.org/iderudit/008171ar

DOI : https://doi.org/10.7202/008171ar

Aller au sommaire du numéro

Éditeur(s)

Presses de l'Université du Québec

ISSN

0226-9554 (imprimé)

1710-0305 (numérique)

Découvrir la revue

Citer cet article

Olscamp, M. (2000). Un air de famille. Entre La relève et Refus global : la génération cachée. Tangence, (62), 7-33. https://doi.org/10.7202/008171ar d'utilisation que vous pouvez consulter en ligne.

https://apropos.erudit.org/fr/usagers/politique-dutilisation/ 


\section{Un air de famille. Entre La relève et Refus global: la génération cachée Marcel Olscamp, Université McGill}

L'objet de cette étude n'est pas constitué d'une "famille" au sens strict, ni même d'une confrérie, puisque les personnes dont il sera question n'ont jamais vraiment revendiqué leur appartenance à un mouvement commun; si ces artistes ont tous étudié au même collège à la même époque, on ne peut non plus utiliser à leur propos le terme de "génération intellectuelle" (au sens où l'entend Jean-François Sirinelli ${ }^{1}$ ), puisqu'il n'est pas dans mon intention de procéder à l'analyse exhaustive d'une cohorte scolaire. Né autour de 1920, le groupe se différencie aussi, par l'âge, de celui retenu par Catherine Pomeyrols dans son ouvrage sur les intellectuels québécois de l'entre-deux-guerres ${ }^{2}$. Disons simplement, même si la notion ne semble guère opérationnelle à prime abord, que j'ai voulu me pencher sur le sort d'une constellation de penseurs et de créateurs que réunit une même vision de la culture. Ce terme a pour précieux avantage de mettre en lumière la ressemblance des individus entre eux sans nier pour autant leur très forte individualité. Solitaires de nature, mais apparentés de façon diffuse par l'esthétique de leurs œuvres, ces créateurs ont respiré dans leur jeunesse le même air du temps et partagé les mêmes goûts et déplaisirs; c'est en ce sens qu'on peut leur reconnaître une indéniable ressemblance...

\section{Le paradigme de l'inquiétude}

Le groupe qui m'intéresse est constitué de jeunes gens formés au Collège Jean-de-Brébeuf, de Montréal, entre 1930 et 1940; il comporte un certain nombre d'écrivains et d'intellectuels connus, parmi lesquels on peut remarquer, entre autres, Jacques Ferron,

1 Jean-François Sirinelli, Génération intellectuelle. Khâgneux et Normaliens dans l'entre-deux-guerres, Paris, PUF, "Quadrige, 160", 1994, 720 p.

2 Catherine Pomeyrols, Les intellectuels québécois: formation et engagements 1919-1939, Paris, Montréal, l'Harmattan, coll. "Le monde nord-américain", 1996, p. 54. 
8

Paul Toupin, Pierre Trottier, Jean-Charles Falardeau, Guy Frégault, Pierre de Grandpré, Pierre Angers, Jacques Lavigne. On y rencontre aussi quelques peintres, sculpteurs et musiciens comme Pierre Mercure, Louis Archambault ou Raymond Daveluy. Mais cette liste est incomplète et les catégories proposées ne sont pas nécessairement exclusives: ainsi Roger Rolland, qui fut d'abord poète, s'intéressera également à la radio et à la théorie littéraire; Guy Dufresne, dramaturge, fera aussi carrière à la télévision. D'autre part, les anciens de Brébeuf s'adonneront à diverses collaborations ponctuelles et interdisciplinaires, selon leurs champs d'intérêt respectifs: Carl Dubuc publie-t-il son recueil de poèmes Jazz vers l'infini? il le fait respectivement préfacer et illustrer par ses condisciples Pierre Vadeboncour et Gabriel Filion. Pierre Baillargeon veut-il publier un album de chansons françaises? il fait appel à la collaboration de ses camarades de collège, le compositeur Jean Papineau-Couture et le peintre Jacques de Tonnancour. Nous avons affaire, comme on le verra, à un groupe qui se perçoit dans une continuité artistique tout en manifestant une rupture silencieuse avec la génération précédente, à la manière de jeunes adultes qui s'émancipent, sans trop de heurts, de la tutelle parentale. Ce réseau informel d'amitiés et d'influences s'apparente un peu, à bien y penser, aux "pléiades" littéraires d'autrefois: il s'étendra dans toutes les sphères de la culture québécoise et finira par contribuer à l'avènement de la Révolution tranquille.

Ces anciens élèves des jésuites, qui parviendront à leur maturité vers 1960, ont pour première caractéristique commune d'avoir vécu leur adolescence entre deux inquiétudes. La première d'entre elles fut causée par la Crise de 1929; ils ont pu en constater de visu les effets sur la société montréalaise de l'époque. Quant à la seconde, engendrée par la menace de la guerre, elle se traduira surtout pour ces jeunes gens par la sombre perspective de la conscription générale au début des années 1940. Deux ouvrages aux titres opportunément évocateurs, publiés à près de vingt ans de distance par un professeur de Brébeuf et par l'un de ses anciens élèves, symbolisent bien l'impression durable que ce climat d'anxiété a laissé sur leur imaginaire: il s'agit de Leur inquiétude, de François Hertel (1936), et de L'inquiétude bumaine, de Jacques Lavigne (1953). Indépendamment de leur contenu manifeste, qui diffère considérablement, ces deux livres apparaissent comme les symptômes d'un tourment qui traversa toute la décennie de 1930. Ce "paradigme" de l'inquiétude était d'ailleurs com- 
mun à toute la jeunesse; on peut en retrouver la source dans l'œuvre de certains penseurs français, comme Daniel-Rops, qui influença aussi, au Québec, les animateurs de La relève.

\section{La mobilisation morale}

Pour mieux comprendre ce climat d'incertitude et avoir une idée du "terreau" intellectuel au sein duquel furent formées les personnalités qui nous intéressent, il faut consulter le journal du collège durant la période de l'entre-deux-guerres. Le Brébeuf c'est son titre - nous révèle, par ce qu'il approuve ou dénonce tout autant que par ce qu'il tait, les enjeux intellectuels, sociaux et littéraires importants de la décennie. Fondé en 1934, à la demande expresse des collégiens - mais fermement supervisé par les professeurs - ce périodique véhicule un discours assez austère qui laisse peu de place à la frivolité. En cette période de crise généralisée, le temps n'est plus aux vaines carabinades, et les jeunes rédacteurs reconnaissent, comme leurs aînés, l'urgence de procéder rapidement à des réformes radicales qu'ils réclament de tous leurs voux. Naguère insouciants, les adolescents prennent soudainement conscience des immenses difficultés que rencontre la plus grande partie de la population de leur pays; du coup, ils en ressentent de la culpabilité: "Nous ne connaissons pas le monde ni la situation des ouvriers et nous ne voulons pas les connaître. Nous croupissons dans une ignorance crasse [...]" confesse un élève. "Si vous désirez aider au relèvement de la classe prolétaire [...], vous devrez, ne l'oubliez pas, descendre de votre tour d'ivoire dans la rue, à l'usine, là où le peuple vit, où il travaille, où il peine ", suggère un autre. En 1936, la parution de Mesure de notre taille, essai de Victor Barbeau qui dénonce le piètre état de l'économie canadienne-française, aura un énorme retentissement dans les institutions d'enseignement du pays. "Je sais [...] que notre peuple s'anémie, qu'il se pervertit, qu'il se gangrène, écrit le lucide essayiste. Ce que je cherche et ne trouve point, ce sont nos œuvres de $v^{5}{ }^{5}$." L'ouvrage agit comme un

3 Jacques Duquette, "Ouvrons les yeux", Brébeuf, Montréal, vol. V, n ${ }^{\text {os }}$ 2-3-4, 13 novembre 1937 , p. 1.

4 Jean Richer, "Devons-nous devenir plus matérialistes?", Brébeuf, vol. IV, no 5,21 novembre 1936, p. 1.

5 Victor Barbeau, Mesure de notre taille, Montréal, imprimé au "Devoir", 1936, p. 14. 
véritable coup de fouet sur la pensée de ces adolescents pétris d'humanités. "La crise nous a réveillés, placés devant une foule de tristes et incontestables faits dont le livre de Monsieur Barbeau est la synthèse ${ }^{6}$ ", constate un journaliste en herbe. "Lisez-moi ça! Vous entendez? d'un couvert [sic] à l'autre, ordonne, péremptoire, un autre zélateur enthousiaste. Tout. Gros texte, petit texte, notes, parenthèses. Tout y est important. Lisez ${ }^{7}$ !"

Durant ces années sombres, les réflexes de culpabilité sont encore très puissants dans cette société profondément religieuse - et donc dans la plupart des collèges du pays. On croit que la crise mondiale plonge ses racines dans l'imperfection foncière de l'être humain: les moeurs corrompues, le matérialisme à outrance, l'absence de charité sont identifiés comme les causes principales du chômage et de la misère. Cette tendance à l'auto-accusation explique la popularité grandissante des organisations d'action catholique, vouées à l'amélioration de la société par le perfectionnement moral de chacun. "L'apostolat de l'étudiant se fait par l'étudiant, et petit à petit c'est la masse qui est atteinte ${ }^{8}$ ", affirme le père Recteur du collège, dans l'une de ses allocutions fidèlement commentée par un apprenti-reporter du Brébeuf. Les autorités religieuses tentent de rénover l'image du catholicisme et de lui faire adopter — bien maladroitement parfois - un langage plus proche de celui de la jeunesse:

Vous retardez, Monsieur le laïc, c'est vous qui "datez"! aujourd'hui, voyez-vous, il y a toute une génération de jeunes collégiens qui ont appris la langue catholique [...], langue claire et claironnante, par quoi se manifestent des âmes claires, les âmes des jeunes collégiens vingtième siècle ${ }^{9}$.

Beaucoup d'adolescents se laissent séduire par cette nouvelle rhétorique attrayante et adhèrent au mouvement de la Jeunesse étudiante catholique (JEC). Ils tentent par la suite, avec beaucoup d'insistance, de mobiliser à leur tour leurs camarades, dans un processus d'émulation qui tient parfois de l'enrôlement! Les collégiens sont sommés, par leurs condisciples les plus convaincus, de

6 Pierre Brais, "En marge de la thèse Barbeau ", Brébeuf, vol. IV, nos 3-4, 24 octobre 1936, p. 4.

7 Jean Filion, "Sur le même ton", Brébeuf, vol. III, n 13, 2 mai 1936, p. 2.

8 Arthur Simard, "Esprit collégial", Brébeuf, vol. II, n 13, 16 avril 1935, p. 1.

9 Brébeuf [pseud.], "Trop catholique", Brébeuf, vol. III, n ${ }^{\text {os }}$ 9-10-11, 15 février 1936, p. 2. 
participer, avec toute la fougue de leur âge, au grand défi que représente le redressement spirituel du Canada français :

Au lieu de nous laisser aller à la déchéance religieuse et nationale, remontons la pente, remontons vers les sommets et la gloire. Que nos visages soient plus brillants de bonté, de force et de sagesse; que tout en nous se hausse et s'affermisse, jusqu'à l'objectif qui seul est vraiment richesse et bonheur: la vertu, la perfection du chrétien ${ }^{10}$.

On le devine, de telles préoccupations, de telles exigences laissent peu de place à la rêverie artistique et aux valeurs de l'imaginaire. Les mouvements d'Action catholique s'inscrivent dans une logique de participation universelle qui ne convient pas nécessairement aux tempéraments solitaires. Tous les collégiens n'adhèrent pas, sur ce point, à l'orientation du journal, comme le déplore un partisan de la JEC scandalisé par des réflexions désobligeantes entendues dans les couloirs de son alma mater: "Le Brébeuf devient plat. Il est trop catholique!" C'est textuel! C'est comme ça qu'on l'a dit! [...] alors pour vous, "catholique" est synonyme de plat, ennuyeux, monotone ${ }^{11}$ ?"

\section{Une dissidence tolérée}

Ce commentaire outré est utile, puisqu'il laisse sous-entendre que certains "Brébeuvois" faisaient mine de prendre leurs distances face à la rhétorique de l'Action catholique. Or, dans la mesure même où ce discours militant, dans les années 1930, se faisait insistant — parfois même tonitruant —, la résistance, elle, devait se montrer plus modérée, car dans cette étrange dynamique d'engagement moral et de professions de foi publiques le seul fait de se tenir en retrait pouvait paraître suspect. C'est précisément ce caractère discret qui marquera l'action des artistes et écrivains formés au collège Jean-de-Brébeuf durant cette période. Derrière les exhortations des jécistes, on peut d'ailleurs déceler, comme à l'envers d'une toile, des phobies et censures sociales - pour reprendre la terminologie de Marc Angenot ${ }^{12}$ - qui trahissent la

10 Marc-André Blain, "Action nationale des jeunes", Brébeuf, vol. II, nos 14-15, 15 mai 1935, p. 8.

11 Brébeuf [pseud.], "Trop catholique", loc cit., p. 2.

12 Marc Angenot, "Le discours social: problématique d'ensemble", Cabiers de recherche sociologique, Montréal, vol. 2, n 1, avril 1984, p. 29. Sur le dis- 
présence de légères dissonances au sein même du collège. C'est une divergence plutôt timide et difficile à évaluer puisqu'elle relève de l'impensable, et donc de l'informulé. On peut toutefois en déceler des traces, en creux, dans le journal des élèves. Il est aussi possible d'identifier, à travers cette parole unanime, quelques éléments, en apparence inoffensifs, qui seront bientôt porteurs d'avenir.

Rendons d'abord à César ce qui appartient à César: les pères jésuites, pédagogues réputés, ne craignaient pas d'exposer leurs élèves à la culture vivante, et nombreux sont les anciens de Brébeuf qui seront toujours reconnaissants à ces parents intellectuels de leur avoir à ce point éveillé l'esprit: "Les jésuites, au niveau collégial, loin de me fermer à la pensée et aux penseurs, à la littérature et aux arts, m'ont au contraire ouvert à ces choses-là, comme ils m'ouvraient à la poésie, ainsi qu'à des valeurs qui me resteraient ${ }^{13}$ ", écrit par exemple Pierre Vadeboncœur. Singulièrement, la peinture moderne et son enseignement entraient pour une bonne part dans cette remise à jour de la culture. Les professeurs avaient par exemple recruté le peintre Maurice Gagnon, ancien étudiant de la Sorbonne, qui venait dispenser au "collège de la montagne" des cours hors-programme sur les artistes contemporains. Une centaine de collégiens s'inscrirent - sur un total possible d'environ quatre cents ${ }^{14}$. C'est sans doute par l'intermédiaire de Gagnon que certains d'entre eux purent faire la connaissance d'Alfred Pellan, de retour d'Europe au début de la guerre. Aussi tôt qu'en 1940, des élèves furent confrontés à son œuvre singulière et à celle des autres peintres modernes, comme en témoigne une série de longs articles fouillés dans le Brébeuf ${ }^{15}$. Ces initiations sont à l'origine de vocations artistiques ou "critiques" chez des gens comme Jacques de Tonnancour, Guy Viau et Pierre Vadeboncœur.

cours so cial de l'entre-deux-guerres, voir mon article intitulé "Le Québec des années trente", Écrits du Canada français, Montréal, n 77, 1993, p. 135161.

13 Pierre Vadeboncœur, "Le Québec expliqué aux Anglais", dans Paul-Émile Roy, Pierre Vadeboncour. Un homme attentif, Laval, Éditions du Méridien, 1995 , p. 72.

14 [Anonyme], "Les cours d'histoire de l'art", Brébeuf, vol. III, n 1, 12 octobre 1935 , p. 2.

15 On peut noter, en particulier, une fine analyse intitulée "Pellan" et signée Jean-Louis Langlois, "élève de philosophie" (Brébeuf, vol. VIII, n 3, 22 novembre 1940, p. 3). 
L'intérêt porté par les collégiens à la musique constitue aussi l'une des manifestations les plus claires de leur fascination pour la création artistique contemporaine. C'est en effet par le biais des disques et des concerts que les adolescents ont le plus ouvertement accès à une certaine actualité esthétique. Sous le regard bienveillant de leurs maîtres, qui encouragent la culture musicale, les élèves bénéficient de multiples activités qui sont suivies avec beaucoup d'enthousiasme: concerts symphoniques à l'Auditorium du Plateau, récitals, "académies musicales" pour les pensionnaires, cercles plus ou moins officiels d'amateurs de disques, etc. Ils découvrent les compositeurs du $\mathrm{xx}^{\mathrm{e}}$ siècle: Bartok, Stravinski, tout un univers sonore qui constitue pour eux une bouffée d'air frais. "Heureusement, écrit un mélomane, les auteurs modernes, artisans de la rénovation musicale française: Debussy, Ravel, Dukas, D’Indy, Fauré, prennent de plus en plus une place importante méritée dans les récitals et concerts, chez nous ${ }^{16}$." Le grand Wilfrid Pelletier fait déjà figure de héros parmi ces jeunes: le Moestro n'a pas sitôt informé le public de difficultés financières que connaissent ses Matinées symphoniques que l'équipe du Brébeuf décide de voler à son secours en lui consacrant plusieurs articles élogieux ${ }^{17}$.

Une partie de cet intérêt renouvelé vient de la démocratisation musicale apportée dans les foyers québécois par la radiophonie. On sait l'importance que ce véhicule d'information et de culture avait déjà pour le groupe de La relève: relisons les écrits de SaintDenys Garneau, qui témoignent des séances d'audition de concerts radiophoniques auxquelles s'adonnaient le poète et ses amis. À la fin des années 1930, le phénomène était encore relativement récent et suscitait des débats houleux dans la presse quant à sa véritable valeur. Même François Hertel, encore professeur à Brébeuf en 1937, trouve à s'inquiéter du fait que Robert Choquette, l'un de nos meilleurs poètes, s'abaisse à rédiger le radio-roman Le curé de village et "délaisse la poésie pour l'émission radiophonique ${ }^{18}$ ".

Les pages du journal étudiant font d'ailleurs écho à ces discussions et donnent à lire plusieurs articles sur ce nouvel appareil

16 Robert Ouellette, "Musique s'il vous plaît", Brébeuf, vol. II, no 9, 16 février 1935, p. 4.

17 La Rédaction, "De la musique", Brébeuf, vol. VII, n 2, 11 novembre 1939, p. 6.

18 François Hertel, "La crise des créateurs ou la trahison de nos clercs", Le Mauricien, Trois-Rivières, vol. I, n 11 , octobre 1937, p. 12. 
14

qui occupe une place grandissante dans la vie quotidienne des Québécois. Le dernier carré de résistants proteste, entre autres, contre la chanson populaire et le jazz, musiques d'enfer que l'on oppose aux grands airs du répertoire. Déjà, en 1929, André Laurendeau trouvait à redire contre le fléau sonore américain qui n'avait "aucune valeur en lui-même ${ }^{19}$ "; avec la généralisation de la radio, qui fait pénétrer la musique dans les foyers, la polémique devient encore plus aiguë et les élèves de Brébeuf y participent aussi: "Il vaut surtout mieux écouter un programme de musique sérieuse que toutes les insanités et les lubricités du jazz, de Lucienne Boyer, de la Palma [...]. Je connais des maisons où le radio est ouvert $[\mathrm{sic}]$ à la journée sans qu'on change de poste une seule fois ${ }^{20}$." Cette méfiance et ces luttes d'arrière-garde auront cependant peu d'effet. Nombre de collégiens - Guy Dufresne, Edmond Labelle, Roger Rolland et plusieurs autres - seront même happés, au sortir de leurs études, par ce médium en pleine expansion, qui réclamait toujours plus de forces vives, constituant de ce fait une manne inespérée pour les créateurs et les artistes du Canada français. Les diplômés de Brébeuf ont fourni un important contingent de professionnels à la radio, devenant ainsi la première génération "audio-visuelle" du Québec...

\section{La littérature pure}

Si la musique et la peinture ont entraîné une transformation plus visible des mentalités étudiantes, c'est à travers la littérature qu'on pourra vraiment prendre la mesure de la véritable évolution des idées. La toile de fond des débats littéraires du Brébeuf trouve son origine dans des polémiques européennes qui avaient récemment retenu l'attention des milieux littéraires montréalais. Ces discussions tournaient autour de la nécessité ou non, pour les écrivains, d'aborder des questions qui ne soient pas exclusivement esthétiques; on peut en résumer les principaux enjeux en invoquant la notion de pureté littéraire, qui circule de façon plus ou moins explicite dans les écrits du temps. L'abbé Henri Bremond, critique parisien, avait publié en 1926 un essai intitulé La poésie pure, qui semble avoir eu quelque influence parmi les

19 André Laurendeau, cité par Catherine Pomeyrols, op. cit., p. 203.

20 Jean Couture, "À propos de musique", Brébeuf, vol. IV, n ${ }^{\text {os }} 1-2,3$ octobre 1936, p. 6. 
critiques québécois; l'aimable académicien y dissertait de la nature ineffable de la beauté poétique et du sens aléatoire qu'il fallait lui donner: "Tout poème doit son caractère proprement poétique à la présence, au rayonnement, à l'action transformante et unifiante d'une réalité mystérieuse que nous appelons poésie pu$\mathrm{re}^{21}$." Un autre essayiste français, Julien Benda, était l'auteur d'un ouvrage aujourd'hui devenu célèbre, La trahison des clercs, qui traitait, sous un autre angle, des mêmes questions de spécificité littéraire en dénonçant l'attitude des intellectuels contemporains, "ces hommes dont la fonction était de contrarier le réalisme des peuples et qui, de tout leur pouvoir et en pleine décision, ont travaillé à l'exciter ${ }^{22}$ ".

Dans le contexte québécois, où la controverse sur la "nationalisation" de la littérature se poursuivait sporadiquement, sous une forme ou une autre, depuis quelques décennies, on pouvait s'attendre à ce que les écrivains soient sensibles aux questions soulevées par ces deux critiques. La vieille querelle reprit donc, actualisée, avec un vocabulaire remis au goût du jour. François Hertel publie en 1937 un article intitulé "La crise des créateurs ou La trahison de nos clercs "; ce titre fait clairement référence à l'essai précité de Julien Benda, auteur qui, incidemment, était venu à Montréal donner une conférence à l'Alliance français ${ }^{23}$. Des affirmations antinationalistes comme la suivante, tirée de La trabison des clercs, avaient certainement heurté les convictions de Hertel, toujours homme d'Église à cette époque et auteur, ne l'oublions pas, d'un roman à thèse destiné à inciter les élèves à se pencher sur les "problèmes nationaux":

Cette adhésion à la passion nationale est singulièrement remarquable chez ceux que j'appellerai les clercs par excellence, j'entends les hommes d'Église. Non seulement l'immense majorité de ces hommes ont [...] adhéré au sentiment national et donc cessé de donner au monde le spectacle de cours uniquement occupés de Dieu, mais ils paraissent bien adopter ce sentiment avec la même passion que [...] les gens de lettres et être prêts,

21 Henri Bremond, La poésie pure, avec Un débat sur la poésie par Robert de Souza, Paris, Bernard Grasset, 1926, p. 16.

22 Julien Benda, La trabison des clercs, Paris, Pauvert, coll. "Libertés", n 25 , [1927] 1965, p. 134.

23 Cette conférence avait d'ailleurs suscité quelques remous; voir à ce propos l'article de Berthelot Brunet: "J'écoute monsieur Benda", Les idées, Montréal, vol. III, n 3, mars 1936, p. 189-192. 
eux aussi, à soutenir leur pays dans ses moins discutables injustices $^{24}$.

Hertel, dans son article, montre qu'il a été profondément ébranlé par le livre de Benda, au point où il pose, à propos des lettres canadiennes, des questions analogues à celles soulevées par La trahison des clercs. Toutefois, il déplace un peu le problème en dénonçant, non pas le nationalisme, comme le fait Benda, mais bien le trop grand nombre de thèses et d'ouvrages utilitaires "d'ordre économique, social, politique" qui se publient à Montréal. "Ces dernières années, la littérature canadienne-française, déjà si pauvre, accuse un recul alarmant. Nos écrivains se détournent de la création pour se perdre dans l'action ou l'enseignement". Lui qui, quelque temps plus tôt, avait cru nécessaire de rédiger un essai pour combattre le sentiment d'inquiétude des adolescents d'aujourd'hui, voici qu'il se réclame à son tour de la pureté littéraire:

Seule vit et survit la littérature pure. J'entends par là celle qui a pour but premier de plaire, d'enchanter. [...] Pour se convaincre de la solidité des ouvres de littérature pure, quand elles sont réussies, on n'a qu'à songer à ce qui reste des littératures latines et grecques. Presque toute la polémique est morte. Le poème épique, le théâtre, le lyrisme, la philosophie pure demeurent éternels ${ }^{25}$.

Malgré cette profession de foi envers les genres littéraires éprouvés, le professeur ne pouvait balayer d'un revers de main ses convictions patriotiques, inscrites de longue date dans ses œuvres et dans sa pensée; on notera d'ailleurs que son article fut publié dans le très régionaliste Mauricien de son ami Clément Marchand. Hertel tentera donc de détourner à son avantage le sens de la littérature pure en lui faisant dire le contraire de ce que Benda entendait lui faire signifier, en l'assimilant à un patriotisme littéraire de bon aloi qui "consiste à intensifier dans nos lettres l'apport spécifiquement canadien tout en continuant de puiser en France la langue avec toute sa pureté, la pensée forte, la sève, le jaillissement français ${ }^{26}$ ".

Ces questions actuelles, on le devine, trouvaient largement leur écho dans les pages du Brébeuf, surtout quand on sait que

24 Julien Benda, La trabison des clercs, op. cit., p. 48.

25 François Hertel, "La crise des créateurs ou la trahison de nos clercs", loc. cit., p. 12.

26 Ibid., p. 13. 
Rodolphe Dubé, s.j. - alias François Hertel — enseignait au collège durant cette période, et que son influence fut décisive auprès de plusieurs. Le fait de s'occuper exclusivement de littérature était presque perçu comme un affront par ceux des rédacteurs qui exigeaient la collaboration de tous au relèvement moral de la société canadienne. Dans le journal des élèves, en tout cas, on ne se prive pas pour ridiculiser, avec quelque condescendance, la pureté littéraire ou poétique, qu'on assimile à de la haute voltige intellectuelle et à un esthétisme désincarné. Le principal reproche qu'on adresse aux condisciples "déviants" — et donc, de mon point de vue, le signe annonciateur d'une évolution possible est une accusation de snobisme et de narcissisme littéraire. Un jeune auteur dénonce avec force "ces intellectuels purs, ceux qui se renferment dans un individualisme farouche contribuant, d'après eux, à se former une personnalité plus marquée, transcendantale ${ }^{27}$ ". Un autre accuse ces esthètes de mépriser ouvertement la faible éducation de leurs compatriotes :

Ils ne délaissent les hautes sphères de la métaphysique où ils planent orgueilleusement dissertant sur la nature intime des choses, sur l'idéal et sur la haute esthétique, que pour regarder avec pitié le pauvre peuple qui n'est pas assez formé, pas assez intelligent pour comprendre et goûter de telles choses ${ }^{28}$.

Enfin, toujours dans l'esprit apostolique des mouvements de jeunesse, un jéciste admoneste ses camarades récalcitrants parce qu'ils refusent - suprême affront! - de lire les œuvres des auteurs dits "catholiques":

Pour eux, ne doivent penser en catholiques que les saints [...]. D'après leur théorie, Verlaine, dans Sagesse, est un hypocrite; Péguy voulait faire sensation; Le Cardonnel: fantaisie littéraire; Mauriac: licence diplomatique qu'on pardonne à son talent. Même Claudel, peut-être, posa-t-il à l'inspiré29.

27 Jean Richer, "Insignifiance", Brébeuf, vol. IV, nos 9-10, 13 février 1937, p. 1.

28 Antoine Geoffrion, "Snobisme intellectuel ", Brébeuf, vol. I, n 5, 14 juin 1934, p. 2-3.

29 David Gourd, "À propos de sincérité", Brébeuf, vol. III, no 13, 2 mai 1936, p. 3. Dans une lettre à sa sour Madeleine (7 décembre 1936), le jeune Jacques Ferron énumère une liste d'auteurs étrangement semblables: "Je suis plus moderne, en ce que mes lectures, que mes poètes préférés, sont des types de nos temps - Claudel, Péguy, Le Cardonnel, A. de Noailles... ou des symbolistes - Verlaine (le Verlaine de la Sagesse) et Samain... etc". Jacques Ferron, Laisse courir ta plume. Lettres à ses sours 1933-1945, présentation de 
18

On remarquera au passage, dans cette liste d'auteurs recommandés, la présence surprenante de Paul Verlaine; ce poète moralement douteux est évoqué dans la stricte mesure où il est l'auteur d'un recueil qui témoigne de sa conversion chrétienne...

Or, l'apprentissage des lettres, au collège, c'était d'abord la découverte des principaux auteurs du Grand Siècle français: Pascal, Corneille, Racine, Molière, La Rochefoucauld, Descartes, Montesquieu. Si bien que, par un détour ironique, la dissidence émanait parfois de l'intérieur même du cours classique, puisque la pratique et l'étude d'une littérature non mobilisée par les enjeux sociaux apparaissaient comme une sorte de résistance passive aux mots d'ordre de la JEC. Face à l'insistance du discours officiel, qui exigeait rien de moins qu'une conscription morale de tous les instants, les collégiens plus individualistes trouvèrent refuge dans la littérature la plus... littéraire, celle précisément qu'on leur enseignait dans leurs cours de Belles-Lettres. "La culture, comme s'en souvient Pierre Vadeboncœur, quoique resserrée jusqu'au ridicule par quelques générations de clercs et de moralistes, était classique en son fond. La sensibilité répond très bien à un modèle sévère et noble; elle ne s'y sent aucunement à l'étroit ${ }^{30}$." La liberté intellectuelle, en somme, coïncidait avec l'enseignement littéraire, et le goût en fut donné aux étudiants par la famille collégiale; comparées à l'atmosphère parfois étouffante du dehors, la culture et la morale intemporelles transmises par le cursus académique semblaient d'une extrême fraîcheur aux élèves plus sensibles.

\section{Le néo-classicisme montréalais}

Cette nouveauté ne passait guère, pour eux, par le genre romanesque. Ce fait peut sembler surprenant, étant donné l'âge d'or que traversait alors le roman français; comme le signale avec justesse Olivier Rony, "la multiplicité des récits, la liberté des formes, le choix des angles d'attaque du réel, l'éclatement croissant des tech-

Lucie Joubert, édition préparée par Marcel Olscamp, Montréal, Lanctôt éditeur, coll. "Cahiers Jacques-Ferron, 3", 1998, p. 34. Il est assez amusant de voir l'adolescent citer des lectures si sagement catholiques, derrière lesquelles on sent la main ferme des professeurs jésuites guidant ses choix sans en avoir l'air.

30 Pierre Vadeboncour, Les deux royaumes, postface de Réjean Beaudoin, Montréal, Éditions Typo, nouvelle édition revue, corrigée et augmentée, 1993, p. 144. 
niques traditionnelles [...] confèrent à cette floraison romanesque une physionomie singulière et attachante ${ }^{31}$ ». Il faut dire que le roman n'avait pas très bonne presse auprès des autorités collégiales, qui se méfiaient de son effet potentiellement pernicieux; les professeurs exerçaient donc une surveillance particulière à son endroit. L'accès des élèves aux romanciers modernes était contrôlé, dans la mesure du possible, et les auteurs en vogue, lorsque d'aventure on en faisait mention, étaient présentés à travers un filtre précis. André Malraux, de passage à Montréal en avril 1937 pour y prononcer une conférence, est décrit par les pères jésuites, dans leur revue L'Ordre nouveau, comme un vulgaire agitateur communiste: "Félicitons-nous que cet écrivain français, qui ne parle pas l'anglais, n'ait pu trouver dans notre métropole, "la deuxième ville française du monde", un coin français pour y débiter sa prose subversive ${ }^{32}$." Quant au "funeste André Gide", suspect aux yeux des professeurs à cause de ses sympathies communistes tout autant que pour des raisons morales, il fut "démonisé" par plusieurs, y compris par François Hertel qui, dans Leur inquiétude, qualifie son œuvre de charogne parfumée. "Que Dieu préserve notre jeunesse d'un si mauvais maître 33 !" conclut-il, pris d'un saint courroux. À l'instar de Verlaine, cependant, Gide se met brièvement à exister pour les pères jésuites en une seule occasion, au moment où il publie son impitoyable $R e-$ tour de l'URSS (novembre 1936): en critiquant les Russes, il devenait un allié momentané des anti-communistes, qui pouvaient triomphalement brandir son exemple:

M. André Gide, qui s'était fait l'ardent panégyriste des Soviets, a voulu voir de près le pays merveilleux que sa plume avait célébré. Il s'en fut donc en U.R.S.S. Il y passa plusieurs mois. Le voici de retour. Un livre nous apporte son jugement. C'est un désenchantement profond ${ }^{34}$.

Quant aux romans canadiens, inutile de dire que, dans le climat idéologique des collèges, leur caractère souvent rural et

31 Olivier Rony, Les années roman 1919-1939. Anthologie de la critique romanesque dans l'entre-deux-guerres, Paris, Flammarion, 1997, p. 15.

32 [Anonyme], "Malraux à Montréal", L'ordre nouveau, Montréal, $1^{\text {re }}$ année, no 14, 20 avril 1937, p. 3. Malraux s'était heurté à la toute nouvelle "Loi du cadenas" qui venait d'entrer en vigueur.

33 François Hertel, Leur inquiétude, Montréal, Éditions "Jeunesse" A.C.J.C. et Éditions Albert Lévesque, 1936, p. 62.

34 [Anonyme], "La Russie sous son vrai jour ", L'Ordre nouveau, $1^{\text {re }}$ année, no 7 , 5 janvier 1937, p. 2. 
passéiste semblait bien indigeste aux lecteurs adolescents qui ne partageaient pas toujours le goût des élites pour le nationalisme. "Mon ami Pierre [...] a lu Jacques et Marie de Napoléon Bourassa, les romans de Marmette, de Gaspé, de Gérin-Lajoie", écrit encore François Hertel dans Le beau risque. "Sans le savoir, Pierre est déjà pétri de vie canadienne ${ }^{35}$." Avouons que le programme n'avait rien pour séduire de futurs auteurs qui penchaient plutôt vers l'esthétisme mallarméen. Rien de bien nouveau non plus du côté des romanciers contemporains: rappelons que, durant la décennie de 1930, on compte, parmi les ouvres les plus plus importantes, des récits comme Un homme et son péché (1934), Menaud, maître-draveur (1937), Trente arpents (1938) et Au cap Blomidon (1932) de Lionel Groulx. Le roman campagnard pouvait-il sérieusement intéresser ces jeunes esprits urbains, déjà largement exposés à la propagande patriotique? "La littérature, alors, pour moi, c'est la littérature française ${ }^{36}$ ", tranchera plus tard Pierre Vadeboncoeur. Un autre écrivain issu de Brébeuf, Pierre Baillargeon, exprimera rétrospectivement son dédain du roman canadien en déplorant son caractère quasi ecclésiastique:

Jadis tous nos écrivains étaient des avocats, des notaires, des médecins, sinon des prêtres. La plupart étaient des ecclésiastiques. Chaque ordre avait son romancier: Farley, Savard, Groulx, Filion, Dablon, et plusieurs autres. La vocation littéraire semblait devoir se confondre avec la vocation tout court ${ }^{37}$.

Hasardons une hypothèse: les romanciers de l'entre-deux-guerres, qu'ils soient français ou canadiens, avaient comme particularité commune d'être engagés - à divers degrés — dans une cause ou une autre: communisme, anticommunisme, action catholique, agriculturisme. Tous avaient une propension à intervenir sur la place publique et dans les débats sociaux; on peut penser que cette conscription générale des esprits laissait les collégiens assez froids.

Parallèlement à ce désintérêt juvénile, on constate que les savants et les critiques, très présents au cours de cette décennie, avaient acquis - nonobstant ce qu'en pensait le professeur

35 François Hertel, Le beau risque, Montréal et Paris, Fides, $18^{\mathrm{e}}$ mille, édition définitive, 1961, p. 28.

36 Pierre Vadeboncœur, "Le Québec expliqué aux Anglais", op. cit., p. 77.

37 Pierre Baillargeon, Les médisances de Claude Perrin, Montréal, Parizeau, 1945, p. 119. 
Hertel — du prestige auprès des collégiens. J'ai déjà signalé le vaste retentissement suscité par Victor Barbeau avec Mesure de notre taille; à cet ouvrage séminal, il faut aussi ajouter les plaidoyers d'Adrien Pouliot, de Marie-Victorin, d'Esdras Minville et d'Édouard Montpetit en faveur de réformes dans leurs domaines respectifs. Aux yeux des enfants de Brébeuf, le prestige intellectuel passait par ces essayistes, malgré l'intérêt plutôt scientifique de leurs livres :

C'est ainsi que nous avons vraiment compris que vers 1920 ou 1930, à travers la pensée d'Édouard Montpetit, d'Esdras Mainville et de quelques autres, comment la maîtrise du commerce et de l'industrie est indispensable à une nation, à sa vie, à sa créativité, à ses valeurs. Il fallait beaucoup d'humanisme et, pour l'époque, une science très intelligemment mariée à l'humanisme et au sens politique, pour le découvrir ${ }^{38}$.

Grâce à ces austères prosateurs, qui abordaient de front les réalités les plus dures et se chargeaient de réclamer de nécessaires améliorations socio-économiques, la "vraie" littérature était sauve : conservant ses prérogatives, elle pouvait enfin devenir une activité essentiellement esthétique et les sphères d'activité demeuraient clairement délimitées. Dans le même ordre d'idées, on peut aussi évoquer les figures de Marcel Dugas, Maurice Hébert, Roger Dantin et Albert Pelletier: indépendamment des idées qu'ils professaient, ces commentateurs littéraires publièrent tous, au cours des années 1930, des recueils de critique fort remarqués. Comme l'a vu Gilles Marcotte, la nouveauté du phénomène tient au fait que ces articles,

destinés à des revues ou des journaux, accèdent si tôt au livre, et que les livres des critiques [ont] un retentissement égal, sinon supérieur, aux œuvres dites de création qui se publient durant les mêmes années. La critique, légitimée par l'édition, [...] devient un genre littéraire conscient de son éminente dignité, qui entend traiter d'égal à égal avec les autres genres ${ }^{39}$.

Du côté de la France contemporaine, une catégorie d'auteurs, qu'on pourrait décrire comme des praticiens d'une littérature "désengagée", retinrent l'attention de certains collégiens de

38 Pierre Vadeboncœur, Gouverner ou disparaître, essais, Montréal, Éditions Typo, nouvelle édition, 1993, p. 136.

39 Gilles Marcotte, "Les années trente: de Monseigneur Camille à la Relève", dans Littérature et circonstances, essais, Montréal, l'Hexagone, 1989, p. 53. 
Brébeuf et leur parurent fraternels; les jeunes Québécois partageaient en cela les goûts du public "hexagonal" cultivé de l'époque. "Quand elle s'aventurait au-delà du tout venant, la consommation culturelle des années 1930 préférait les hommes de lettres affectés aux hommes d'action moins courtois: les décorateurs délicats à la Jean Cocteau ou les aphoristes subtils à la Giraudoux ${ }^{40}$." Ces auteurs à la mode avaient une autre caractéristique fondamentale: ils reproduisaient et réinterprétaient à leur façon les grands thèmes de la littérature classique, ce qui les plaçait dans une lumière immédiatement familière aux élèves montréalais. Ce néo-classicisme rendait par ailleurs leurs écrits esthétiquement inattaquables et constituait en quelque sorte la caution de leur modernité :

Dans l'entre-deux-guerres, [Giraudoux] n'est pas le seul à pratiquer la réécriture au théâtre: Cocteau (Antigone, La Machine infernale), Claudel (Protée), Gide (Edipe) et, parmi des auteurs moins connus, André Obey, ont contribué à remettre à l'honneur les mythes antiques. Or, dans le contexte dramatique de cette époque, ce phénomène n'était pas une simple mode mais se dotait d'une intention esthétique. [...] l'écriture au second degré apparaît comme un moyen de souligner d'entrée la spécificité de l'œuvre dramatique en affichant sa littérarité ${ }^{41}$.

En d'autre termes, ces auteurs, en se reférant à une littérature canonique, affirmaient leur indépendance vis-à-vis de l'actualité et des contingences du monde. Voilà une modernité qui avait tout pour plaire aux collégiens montréalais! Grâce à leur formation humaniste, ces derniers se trouvaient pour une fois en intimité immédiate avec une avant-garde européenne dont ils maîtrisaient les principaux codes; de ce fait, ils se sentaient confortés dans leur décision de ne point adhérer ouvertement aux lourdes idéologies que leur société leur prescrivait par ailleurs. Giraudoux, Cocteau ou Valéry se permettaient de "revisiter" le mythe d'Eedipe ou la Guerre de Troie; quoi de plus rassurant pour des esprits formés par l'étude de ces grands récits universels? Se souvenant de ces exemples haut placés, Paul Toupin pourra plus

40 Eugen Weber, La France des années 30. Tourments et perplexités, traduit de l'anglais par Pierre-Emmanuel Dauzat, Paris, Fayard, 1995, p. 301.

41 Catherine Nier, "Écriture "au second degré" et conception du théâtre ", dans Lise Gauvin (dir.), Jean Giraudoux et l'écriture palimpseste, Montréal, Université de Montréal, Département d'Études françaises, Paragraphes, n 14, 1997, p. 218-219. 
tard rédiger un Brutus tout en ayant l'impression de rester actuel; pour les mêmes raisons, Pierre Baillargeon, en compagnie de ses condisciples Jean Papineau-Couture et Jacques de Tonnancour, publiera sans remords un joli cahier d'Églogues virgiliennes. Même Jacques Ferron, à la manière de Giraudoux et de Cocteau, ne pourra s'empêcher de réinterpréter à son tour les classiques dans ses premières pièces de théâtre.

\section{La filière valéryenne}

Parmi ces contemporains français notables, la figure de Paul Valéry s'impose d'une façon toute spéciale. J'ai montré ailleurs ${ }^{42}$ comment son œuvre avait pu marquer profondément celle de Jacques Ferron, au point où, écrit ce dernier, la magie valéryenne l'avait ensorcelé "comme un beau verbe sacré dont la présence sous-jacente rendait sa parole incertaine et dérisoire ${ }^{43}$ ". Durant l'entre-deux-guerres, l'auteur de "La jeune Parque" était au faîte de la gloire; poète rigoureusement respectueux de la prosodie, il dédaignait le genre romanesque et s'exprimait à travers des formes éprouvées. Le dialogue de type platonicien et l'aphorisme, par exemple, qui n'avaient plus de secrets pour lui, étaient loin de dépayser les collégiens de Montréal, déjà rompus aux versions grecques et aux préceptes. L'admiration pour cet écrivain était donc courante parmi les élèves déjà dotés d'une sensibilité littéraire. D'autre part - ce qui ne gâtait rien! - Paul Valéry se sentait bien, politiquement parlant, "dans une démocratie clairement élitiste. D'où son admiration pour les jésuites et Saint-Ignace de Loyola $[\ldots]^{44}$ ". Par conséquent, il est bien compréhensible que les professeurs de Brébeuf aient éprouvé une admiration réciproque pour lui, et qu'ils ne se soient point opposés à la diffusion de ses œuvres parmi les élèves. Que Valéry ait aussi fait preuve d'un relatif intérêt politique pour le Portugal de Salazar n'avait rien non plus pour déplaire aux jésuites; au contraire, il semblait ainsi

42 Le fils du notaire. Jacques Ferron 1921-1949. Genèse intellectuelle d'un écrivain, Montréal, Fides, 1997; voir en particulier p. 178-181 et 212-213.

43 Jacques Ferron, La charrette, préface de Ginette Michaud, avec la collaboration de Patrick Poirier pour les notes et l'établissement du texte, Montréal, Biblithèque québécoise, 1994, p. 97.

44 Monique Allain-Castrillo, "Clio ineffaçable. Le relief du politique valéryen sur fond d'Espagne", dans Serge Bourjea (dir.), Paul Valéry et le politique, Paris, l'Harmattan, 1994, p. 196. 
mener le même combat que l'École sociale populaire, organisme créé par la Compagnie de Jésus pour défendre les principes du corporatisme social.

On n'a pas encore pris toute la mesure de l'ascendant que Valéry a pu avoir sur les intellectuels québécois, sans doute parce qu'il était dans la nature même de cette influence de se manifester avec parcimonie; la logique de la pensée valéryenne décourage en effet les débordements d'enthousiasme et le prosélytisme. Il n'en reste pas moins que beaucoup de collégiens se sont sentis interpellés par cette œuvre altière, suffisamment en tout cas pour que l'on soit tenté d'y voir un fil conducteur, surtout à partir des années 1940. C'est une filiation bien vivante, mais discrète, qui se répand surtout au Québec grâce à l'intercession fervente des écrivains de Brébeuf. La citation suivante, tirée d'un recueil de souvenirs de Paul Toupin, pourrait tout aussi bien avoir été recueillie sous la signature de Pierre Trottier, de Jacques Ferron ou de Jacques Lavigne:

Des écrivains que j'ai pratiqués, Valéry occupe une place à part. Je l'ai lu dès le collège avec une admiration sans réserve. Sa réputation d'écrivain hermétique n'était pas une objection sérieuse. Il était l'auteur le plus moderne et le plus classique à la fois. Sa poésie me paraissait parfaite, autant que sa prose. Il n'y avait pas de style plus rigoureux ${ }^{45}$.

L'œuvre de Valéry fut d'abord introduite au collège par Pierre Baillargeon. Doué d'une vaste culture et d'une profonde érudition, cet homme de lettres précoce était devenu l'ami de ses professeurs, avec lesquels il s'entretenait fréquemment d'art et de littérature. L'un d'entre eux, le Père Robert Bernier, fondait de grands espoirs sur son ancien élève, en qui il voyait un jumeau canadien du poète de Sète:

On dit bien que le milieu est un plus puissant éducateur que l'école, lui écrit-il en janvier 1939. Pensez à ce que votre œuvre ferait pour le nôtre. Aidez-nous à moins rougir de nous-mêmes. Vous vous devez, pour vos années de classe, au moins des "Rhumbs" ${ }^{46}$ !

45 Paul Toupin, Au commencement était le souvenir, Montréal, Fides, coll. "Nénuphar", 1973, p. 65-66.

46 Pierre Bernier à Pierre Baillargeon, lettre, 12 janvier 1939. Je remercie vivement madame Mireille Baillargeon de m'avoir laissé consulter la correspondance et le journal inédits de son père. Le rhumb est une unité de mesure 
En classe de Philosophie, Baillargeon avait composé quelques pièces clairement imitées de Valéry: ce dernier ayant par exemple publié un poème intitulé "L'insinuant", son émule montréalais fait paraître, dans le journal des élèves, "L'insidieux", texte portant le sous-titre savamment négligé — et tout aussi valéryen de "Poème abandonné ${ }^{47}$ ". Influencé à la fois par le "Narcisse parle" de l'auteur méditerranéen et par les tragédies de Racine, Baillargeon commet un "Bérénice parle" qui réalise un curieux syncrétisme entre le labeur scolaire de l'apprenti et le style aérien de son maître à penser ${ }^{48}$. Paul Toupin, condisciple de Baillargeon, considère que cette vénération active et partagée pour Valéry fut la condition même de leur carrières littéraires respectives :

Au collège que j'avais fréquenté ici, notre classe était divisée en claudéliens et valéryens, Pierre et moi étions valéryens. Il y avait également des mauriaciens, des bernanosiens et je me demande s'il faut voir un signe dans ceci que de nous tous, seuls Baillargeon et moi sommes devenus écrivains ${ }^{49}$.

En 1939, Baillargeon séjournera à Paris afin d'y poursuivre de vagues études de médecine; il les abandonnera bientôt pour se mêler à la diaspora littéraire canadienne et, surtout, pour assister aux leçons très courues de son idole littéraire au Collège de France. Il osera même envoyer quelques-uns de ses poèmes à l'illustre poète, qui eut semble-t-il des bons mots à son endroit ${ }^{50}$. Baillargeon se préparait à mettre sur pied, à son retour au Canada, une revue littéraire, Amérique française, dont l'élégante facture et l'éclectisme ressembleront à s'y méprendre à ceux de la revue trimestrielle Commerce, que dirigeait Valéry en France. Et comme si ces rapprochements ne suffisaient pas, l'écrivain montréalais aura la bonne idée, en 1948, d'intituler son deuxième livre Commerce! On ne s'étonnera pas que Baillargeon, apprenant la mort de

maritime; Valéry, poète méditerranéen, a utilisé ce mot rare comme titre de l'un de ses ouvrages.

47 Pierre Baillargeon, "L'insidieux. Poème abandonné", Brébeuf, vol. IV, no 2, 24 octobre 1936, [p. 7].

48 Pierre Baillargeon, "Bérénice parle", Brébeuf, vol. IV, nos 6-7-8, 21 décembre 1936, [p. 8].

49 Paul Toupin, cité dans André Gaulin, Entre la neige et le feu. Pierre Baillargeon, écrivain montréalais, Québec, PUL, coll. "Vie des lettres québécoises", $\mathrm{n}^{\circ} 18,1980$, p. 22.

50 Voir, à ce propos, la "Présentation" du P. Bernier au recueil d'essais posthume de Baillargeon, Le choix (Montréal, HMH, coll. "Constantes", no 21, 1969, p. 16-17). 
Valéry en 1945, ait noté dans son journal intime: "Valéry mort. Deuil : le seul dont j'ambitionnais de mériter l'estime ${ }^{51}$."

\section{Autour d'Amérique française}

Amérique française a été fondée en 1941 par Baillargeon, en compagnie d'un autre ancien du collège, Roger Rolland, qui rédigera quelques années plus tard, à Paris, une thèse sur la versification poétique ${ }^{52}$. François Hertel contribua, apparemment, au lancement de ce périodique de belle tenue, auquel collaboreront aussi, tour à tour, la plupart des jeunes intellectuels de Brébeuf. Le journal inédit de Baillageon montre que la revue faillit porter un titre emprunté à Valéry, Rhumbs; Rolland, quant à lui, suggéra un nom à consonnance tout aussi valéryenne, Gueule d'azur. Même François Hertel, "exilé" depuis peu au collège de Sudbury par ordre de ses supérieurs, se réclame du grand écrivain dans une lettre qu'il fait parvenir à son ami : "La bêtise n'est pas mon fort" a dit notre maître. J'ajouterai qu'elle n'est pas "forte" et qu'on finit toujours par la vaincre, pourvu qu'on tienne. Ah! si je parviens à remettre le pied à Montréal, je vous aiderai à "financer" [la revue ${ }^{53}$." Dans ce nom ambitieux d'Amérique française, l'adjectif était plus important que le substantif : il manifeste le désir des fondateurs, en l'absence de tout contact avec la France occupée, de devenir "le point de ralliement de l'élite française de la province, voire même du continent américain ${ }^{54}$ ".

La création d'Amérique française eut lieu dans un esprit de confrontation feutrée, mais réelle, avec La relève, revue émanant de l'autre collège montréalais des jésuites, le Sainte-Marie. Plus tard, Hertel interprétera de façon un peu restrictive les différences entre les deux périodiques: selon lui, il s'agissait d'une émulation naturelle entre les rejetons des deux institutions ${ }^{55}$. En réalité, les

51 Pierre Baillargeon, journal inédit, 25 juillet 1945.

52 Roger Rolland, Poésie et versification. Essai sur la liberté du vers, Montréal, Fides, 1949, $189 \mathrm{p}$.

53 François Hertel à Pierre Baillargeon, lettre, 6 juillet 1941. La phrase citée par Hertel est l'incipit de Monsieur Teste.

54 Jolyne Laplante-Paquet, "Introduction aux idées de la revue Amérique française", mémoire de maîtrise en Études françaises, Université de Montréal, septembre 1973, p. 4.

55 "L'opposition à $\mathrm{La}$ Relève [...] provient tout simplement du fait que La Relève est née d'anciens du Collège Sainte-Marie et Amérique française d'anciens 
divergences de vues furent plus profondes qu'une simple rivalité entre deux groupes apparentés. Même si Baillargeon avait déjà publié dans $L a$ relève, il ne tenait pas ce périodique en bien haute estime, le trouvant exagérément religieux et trop peu littéraire: "Le groupe de La Relève: un groupe de jeunes défroqués par indisposition", écrit-il avec hauteur dans son journal en janvier $1941^{56}$. Quelques mois plus tard, au moment où les animateurs changèrent le nom de la revue, il aura ce commentaire significatif :

[...] il n'y a plus de Relève; c'est maintenant La Nouvelle Relève, 64 pages, des chroniques régulières. Je voulais un nouveau titre. La Nouvelle Relève, ça a l'air d'une vieille fille modernisée. Malgré tout, il n'y a encore que La Relève. La Nouvelle Relève. Après ce sera, je suppose: La dernière nouvelle Relève. Quel chemin de croix. Rien de plus vieux, de plus sec, qu'une revue qui se rebaptise nouvelle ${ }^{57}$.

Après avoir envisagé de collaborer avec les éditeurs de cette revue, Baillargeon décidera de faire cavalier seul en créant luimême une tribune à sa mesure, qui sera aussi le point de ralliement informel de sa génération.

La révolution esthétique, façon Brébeuf, n'a rien d'une rupture fracassante; aussi faut-il plutôt parler ici d'une transformation graduelle, assez lente en apparence, qui crée un maillon essentiel d'une chaîne évolutive. Amérique française incarnera au Québec l'amour sélectif et soigneusement circonscrit d'une image de la France, celle qui respecte les formes artistiques séculaires:

Dans la perspective critique de la revue, l'état de la littérature contemporaine n'est guère reluisant. [...] Une certaine jeunesse renie Valéry et Proust au nom d'André Breton. Contre cette décadence, Amérique française luttera sa vie durant, se posera en objecteur à la vague d'éclectisme et de modernisme, y dénonçant des vices de goûts criants et une dangereuse anarchie des idées et des formes ${ }^{58}$.

La relève et La nouvelle relève, comme le mentionne Gilles Marcotte, ont en leur temps marqué l'entrée du "loup" personnaliste

du Brébeuf ". François Hertel à Jolyne Laplante-Paquet, lettre, 13 mai 1972.

Ibid., p. 230.

56 Pierre Baillargeon, journal inédit, 12 janvier 1941.

57 Pierre Baillargeon, journal inédit, 3 septembre 1941.

58 Jolyne Laplante-Paquet, ibid., p. 45. 
dans la bergerie du catholicisme québécois ${ }^{59}$; Amérique française, par son parti pris résolument littéraire et artistique, offre une occasion au loup de faire un pas de plus à l'intérieur de la bergerie culturelle... Il est extrêment révélateur que les supérieurs de François Hertel aient fini par interdire au philosophe de publier dans la revue qu'il avait lui-même contribué à fonder, comme ce dernier s'en ouvre à Baillargeon dans une lettre de 1942: "Pour le moment, il m'est impossible de vous adresser un texte. Ici, on ne veut plus que je publie dans Amérique française. On n'aime pas la revue. Que voulez-vous que j'y fasse! On parle d'influence malsaine, etc. ${ }^{60}$ ". Malgré les allures sages de ce périodique, sa nouveauté n'échappa donc pas à l'œil exercé des censeurs: sous le couvert d'un néo-classicisme de bon aloi, Baillargeon et ses amis surent faire preuve d'une certaine audace feutrée, encore éloignée des fracas de Refus global, mais néanmoins nécessaire.

Chez ces jeunes écrivains, les traces de la formation reçue au Brébeuf prennent une coloration un peu particulière. On peut généralement dire d'eux qu'ils se conforment aux canons de la langue française: les Pierre Vadeboncour, Carl Dubuc, Paul Toupin, Pierre Baillargeon et Jacques Ferron, pour ne nommer que les plus connus, ne sont pas précisément des expérimentateurs langagiers et des déconstructeurs de syntaxe. Ce respect des traditions s'étend aussi aux genres littéraires pratiqués par les membres de ce groupe qui n'en fut pas un. On retrouve parmi eux des philosophes (Jacques Lavigne, Pierre Angers), des essayistes, des moralistes et des mémorialistes (Dubuc, Viau, Baillargeon, Toupin, mais aussi Pierre de Grandpré et Jean-Charles Falardeau), des dramaturges (Toupin, Guy Dufresne et Ferron) et des poètes (Trottier, Dubuc, d'autres moins connus comme Edmond Labelle). Bref, ce sont des auteurs qui s'adonnent, en gros, à la vraie littérature, celle qui leur fut enseignée avec passion durant leur adolescence. À peu de choses près, les genres qu'ils pratiquent sont ceux-là même que François Hertel identifiait, dans son article de 1937, comme faisant partie de la littérature pure.

59 Gilles Marcotte, "Les années trente: de Monseigneur Camille à la Relève", loc. cit., p. 63.

60 François Hertel à Pierre Baillargeon, lettre, 17 juillet 1942. 


\section{Romanciers malgré eux}

Le collège Brébeuf produisit aussi, durant la période qui nous intéresse, deux romanciers, Pierre Baillargeon et Jacques Ferron; mais ces prosateurs furent tellement atypiques que leur ouvre de fiction illustre, a contrario, le même phénomène: à cause de leur méfiance première pour le genre romanesque, ils contribuèrent paradoxalement à en renouveler la forme au Québec. Le roman, catégorie impure par excellence à leurs yeux, allait leur permettre d'expérimenter, comme en se jouant, de nouvelles structures.

Pierre Baillargeon, en plus de nourrir une véritable admiration pour Paul Valéry, fut toute sa vie un lecteur passionné de Montaigne, de La Rochefoucauld et des moralistes du Grand Siècle. Édifié par ces maîtres, il se faisait une haute idée de la littérature et du métier d'auteur, qui se heurta à l'incompréhension de ses compatriotes et lui fit une réputation de puriste. Étant donné ses goûts d'essayiste et son réel talent pour l'analyse littéraire, on peut se demander pourquoi il opta pour l'écriture de fiction; mais ses quatre "romans", publiés entre 1940 et 1948, se distinguent justement parce que la critique, avec raison, hésite à les classer sous cette catégorie générique. Baillargeon lui-même ne les a d'ailleurs jamais nommément identifiés comme tel: ce sont de brefs récits comportant de vagues embryons d'intrigues placés comme à regret au début de l'ouvrage. L'auteur condescend à se conformer au goût du jour avant de vite oublier ce désagrément pour mieux se consacrer à ses chères idées abstraites.

Les médisances de Claude Perrin, son livre le plus connu, met en scène un narrateur anonyme qui publie les mémoires d'un certain Claude Perrin. En réalité, il s'agit ici d'un recueil d'essais de Pierre Baillargeon sur l'art et la littérature; une fois expédiée l'ébauche d'intrigue, l'auteur se plonge avec soulagement dans sa prose de moraliste, à l'aide de brefs chapitres au cours desquels son alter ego, Perrin, règle ses comptes, en quelques paragraphes lapidaires, avec la société. Tout absorbé par ses argumentations, l'essayiste en oublie presque de maintenir jusqu'au bout les artifices qu'il a lui-même établis! Et que dire de Commerce, son récit suivant? L'ouvrage est si peu romanesque que le critique René Garneau, sensible à son ambiance valéryenne, intitulera son compte rendu de l'œuvre "M. Teste au Canada ${ }^{61}$ ". Le narrateur,

61 René Garneau, "M. Teste au Canada ", Le Canada, Montréal, 14 juillet 1947, p. 5. 
ici, se contente de rapporter des conversations tenues par le même Claude Perrin; l'intrigue de Commerce est donc chronologiquement antérieure aux Médisances. Le personnage est libraire de son métier; bien entendu, "On ne trouvait en montre chez lui que les classiques ${ }^{62}$." Il reçoit quelques amis dans sa boutique et devise aimablement de littérature avec eux. On trouve aussi, dans ce livre qui refuse obstinément de se laisser aller au roman, des dialogues de théâtre, des réponses aux critiques de Baillargeon, des sonnets, des articles d'analyse littéraire, mais surtout des maximes et des aphorismes, à la manière des grands moralistes français et du Paul Valéry de Mélange.

Il faut reconnaître que ces récits, rédigés pour ainsi dire le dos tourné au roman, sont formellement assez novateurs. Baillargeon les a parsemés d'épigrammes qui dénigrent le genre romanesque, rehaussant par le fait même la réputation du classicisme: "Dans les romans modernes, les héros boivent et disent des choses profondes: l'ivresse fait passer le morceau"; "Vivant, appliqué au roman, devient péjoratif comme dans l'expression bon vivant" ; "Qui pardonne au romancier de lui avoir fait lire dix pages pour une pensée?"; "La vie du roman, c'est la tienne que tu perds à le

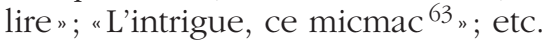

L'autre romancier formé au collège Brébeuf, c'est bien sûr Jacques Ferron. Bien que l'auteur de L'amélanchier semble à première vue moins étranger au roman que Baillargeon - qu'il présente incidemment comme son mentor littéraire -, il partage la même conception exigeante de la littérature. Il professait aussi du moins à ses débuts - le même dédain pour le genre romanesque: "J'aime beaucoup bouquiner. [...] J'aime trouver le classique dans un cloaque de romans ${ }^{64}$ ", écrivait-il déjà à sa sœur Madeleine en avril 1938. On sait qu'il tenta d'abord sa chance comme poète et essayiste dans les pages de journaux étudiants ; il rédigea ensuite un premier récit, mais ne put trouver d'éditeur; c'est alors qu'il entreprit une carrière de dramaturge avant de tenter à nouveau sa chance au début des années soixante.

Les ouvres de Ferron se ressentent aussi de sa formation humaniste, d'abord par l'écriture, ensuite par son recours constant à la

62 Pierre Baillargeon, Commerce, Montréal, Les éditions Variétés, 1947, p. 8.

63 Ibid., passim, p. 145-150.

64 Jacques Ferron, Laisse courir ta plume, op. cit., p. 85. 
mythologie. Comme chez Baillargeon, le roman ferronien ne mérite jamais pleinement cette appellation, puisque l'auteur abandonne rarement son lecteur au simple jeu de l'intrigue. Ses récits sont toujours accompagnés de toute une machinerie - interventions surnaturelles ou fantastiques, digressions, reportages ethnologiques, un peu comme si le romancier, rétif, refusait d'être la dupe de sa propre histoire. Quand ses romans ne sont pas carrément des élaborations fictives construites autour de ses essais, ils sont encadrés par de longs commentaires qui nous expliquent comment il faut comprendre le récit principal; pensons ici aux Roses sauvages, "Petit roman suivi d'une lettre d'amour soigneusement présentée", ou aux Confitures de coings, flanqué d'un "Appendice aux Confitures de coings" qui tente d'en orienter la lecture. Il arrive parfois aussi, comme dans Le ciel de Québec, que le récit ferronien soit interrompu par un intermède qui en brise la linéarité; réminiscences, sans doute, du Cocteau d'Antigone et du Giraudoux d'Électre, auteurs friands de ruptures métathéâtrales et d'emboîtements formels. Ferron est aussi le spécialiste des structures reprises d'une ouvre à l'autre: Cotnoir et Le ciel de Québec sont deux romans qui, chapitre après chapitre, remontent chronologiquement le temps; La nuit, La charrette et Le ciel de Québec, réduits à leur plus simple expression, présentent tous trois la même situation centrale: un Anglais qui se retrouve nuitamment dans un lieu mal famé.

Bref, Baillargeon et Ferron, de par leur formation, ont été des rénovateurs accidentels du roman québécois, à cause de leur désinvolture même et du peu d'estime qu'ils portaient à l'intrigue. Comme il s'agit pour eux d'un genre littéraire mineur, ils s'autorisent à le traiter cavalièrement, à l'utiliser comme un fourre-tout commode; ils le traitent sans aucun ménagement. On peut même penser que la pratique du roman, chez ces fins observateurs de la scène littéraire, n'était pas exempte de considérations stratégiques: les romanciers ne trouvent-ils pas plus rapidement, en général, le chemin de l'édition? C'est dans la mesure où ils méprisent le genre romanesque qu'ils s'autorisent à le rudoyer, à le critiquer de l'intérieur, puisque, laissés à eux-mêmes, ils auraient eu tendance à respecter les structures plus classiques.

\section{Conclusion : Prisme d'yeux}

Mises à part les incartades romanesques de Baillargeon et de Ferron, la "manière" brébeuvoise tient tout entière dans cette 
32

posture littéraire un peu hiératique et dans la retenue formelle; elle se rapproche un peu, à bien y penser, de la "mesure méditerranéenne" que proposera plus tard Albert Camus dans son Homme révolté. Plusieurs anciens de Brébeuf, qui prirent part aux polémiques entourant la peinture dans l'après-guerre, adoptèrent, dans le domaine qui leur était propre, la même attitude modérée: on les retrouve réunis autour d'Alfred Pellan, parmi les signataires de Prisme d'yeux, ce manifeste qui préconisait un équilibre dans les arts. Comme ce texte fut rédigé par un artiste issu de Brébeuf, on y découvre des allusions à la "pureté " artistique et un appel à une peinture débarrassée des considérations autres que picturales:

Prisme d'Yeux s'ouvre à toute peinture d'inspiration et d'expression traditionnelle. Nous pensons à la peinture qui n'obéit qu'à ses plus profonds besoins spirituels dans le respect des aptitudes matérielles de la plastique picturale.

Nous cherchons une peinture libérée de toute contingence de temps et de lieu, d'idéologie restrictive et conçue en dehors de toute ingérence littéraire, politique, philosophique ou autre qui pourrait adultérer l'expression et compromettre sa pureté.

Peinture pure? - Si l'on veut ${ }^{65}$.

Ce texte raisonnable, qui vantait les mérites de l'audace novatrice tempérée par le respect du matériau, est bien dans l'esprit de ce qu'il nous faut désormais appeler la "sensibilité" de Brébeuf, puisque cette famille spirituelle ne fut jamais suffisamment structurée pour qu'il soit possible de parler d'une "école". Sous la signature du principal rédacteur, Jacques de Tonnancour, on retrouvait aussi celles de deux autres artistes formés dans cette institution: le sculpteur Louis Archambault et le peintre Gabriel Filion. Le manifeste aurait pu être contresigné par Pierre Baillargeon, ami et mentor de Tonnancour, qui s'était d'ailleurs empressé, en 1941, de mettre Pellan à contribution pour illustrer les pages d'Amérique française. Le peintre, qui se prononçait au début des années 1940 en faveur d'une évolution progressive de la peinture canadienne, avait trouvé une oreille attentive auprès de ces collégiens.

65 Cité dans Guy Robert, Pellan. Sa vie et son ceuvre, Montréal, Éditions du Centre de psychologie et de pédagogie, coll. "Artistes canadiens", 1963, p. 49. 
On reconnaît bien là la modération si caractéristique des artistes dont nous venons de faire la connaissance. Formés à une époque où l'individualisme et le quant-à-soi suscitaient quelque suspicion, ils ont su exploiter, détourner à leur avantage les principes établis de leur formation, de manière à exprimer une sensibilité nouvelle. Partisans de "l'implosion", ils laissent intactes les apparences et gardent un respect civilisé envers les formes plus anciennes tout en les rénovant de l'intérieur. 